\title{
Intraday Patterns in the Cross-section of Stock Returns
}

\author{
STEVEN L. HESTON, ROBERT A. KORAJCZYK, and RONNIE SADKA*
}

May 3, 2010

\begin{abstract}
Motivated by the literature on investment flows and optimal trading, we examine intraday predictability in the cross-section of stock returns. We find a striking pattern of return continuation at half-hour intervals that are exact multiples of a trading day, and this effect lasts for at least 40 trading days. Volume, order imbalance, volatility, and bid-ask spreads exhibit similar patterns, but do not explain the return patterns. We also show that short-term return reversal is driven by temporary liquidity imbalances lasting less than an hour and bid-ask bounce. Timing trades can reduce execution costs by the equivalent of the effective spread.
\end{abstract}

\footnotetext{
${ }^{*}$ Heston is with the University of Maryland, Korajczyk is with Northwestern University, and Sadka is with Boston College. We thank Joseph Cerniglia, Ian Domowitz, Lisa Goldberg, Campbell Harvey (the editor), Ravi Jagannathan, Bruce Lehmann, Maureen O’Hara, Michael Pagano, Mark Seasholes, Avanidhar Subrahmanyam, Dimitri Vayanos, Julie $\mathrm{Wu}$, the anonymous referees and associate editor, and seminar participants at the NBER Microstructure Meeting; Center for Research in Econometric Analysis of Time Series, Aarhus Universitet; Boston College; Brandeis University; Cornell University; CREST-INSEE, Paris; HEC Montréal; McGill University; Northwestern University; University College Dublin; University of Southern California; the Citigroup Quant Conference; Goldman Sachs; and PanAgora Asset Management for comments. We thank Lew Thorson for computational assistance. We acknowledge financial support from PanAgora Asset Management and Korajczyk acknowledges the financial support of the Zell Center for Risk Research and the Jerome Kenney Fund. Forthcoming in the Journal of Finance.
} 
We postulate that systematic trading and institutional fund flows lead to predictable patterns in trading volume and order imbalances among common stocks. If these patterns are fully anticipated, then they should not cause predictability in stock returns. Yet, we find periodicity in the crosssection of stock returns. To study the nature of intraday periodicity we divide the trading day into 13 half-hour trading intervals. A stock's return over a given trading interval is negatively related to its returns over recent intervals, consistent with the negative autocorrelation induced by bid-ask bounce and lack of resiliency in markets. However, there is a statistically significant positive relation between a stock's return over a given interval and its subsequent returns at daily frequencies (i.e., lags of $13,26,39, \ldots$ periods). That is, knowing that the equity return of XYZ, Inc. is high between 1:30 PM and 2:00 PM today has explanatory power for the return on XYZ, Inc. equity at the same time tomorrow and on subsequent days. This effect is statistically significant for at least 40 trading days.

Two disparate strands of the literature, when taken together, suggest the presence of intraday periodicity in trading volume and order imbalances. First, there is substantial evidence that fund flows to certain types of institutional investors exhibit autocorrelation. Del Guercio and Tkac (2002, Table 5) find that flows to mutual funds exhibit significant autocorrelation while flows to pension fund managers show much weaker autocorrelation. Frazzini and Lamont (2008, Figure 1) show that fund flows into mutual funds in the current quarter are related to fund flows in many future quarters (up to 18 quarters). Lou (2008) also finds evidence of persistent fund flows into and out of mutual funds. Blackburn, Goetzmann, and Ukhov (2007) find that the autocorrelation in mutual fund flows varies by category, with value funds exhibiting greater autocorrelation in flows than growth funds. Even without explicit autocorrelation in fund flows, there may be autocorrelation in trading by a given institutional manager. For example, some managers rebalance individual customers' separate accounts on successive days. Campbell, Ramadorai, and Schwartz (2009) use quarterly 13-F filings and intraday trading data from the New York Stock Exchange's Trade and Quotation (TAQ) database to estimate the aggregate trading behavior of institutional investors at a daily frequency. Their evidence indicates that institutional trading is highly persistent. That is, institutional investment managers tend to buy or sell the same stocks on successive days.

Second, there is a growing literature on trading algorithms that are designed to minimize trading costs or optimize the trade-off between trading costs and the uncertainty associated with the prices obtained over the trading program. For example, Bertsimas and Lo (1998) study the problem of minimizing the expected cost of executing an exogenously specified trade size over an exogenously 
given horizon. In the simplest version of their model the price impact of trade is permanent and proportional to the number of shares traded while non-trade public information causes mean zero asset price changes. The lowest expected impact strategy is to trade an equal number of shares at every point in time. If conditional mean price changes are nonzero, then it would be optimal to either speed up or slow down trading relative to this base case (depending on the expected direction of price movements and the direction of trade).

A number of papers extend the above results to incorporate the risk of the trading program, in addition to the expected trading cost, for risk averse traders (e.g., Almgren and Chriss (2000), Grinold and Kahn (2000), Vayanos (2001), Huberman and Stanzl (2005), and Engle and Ferstenberg (2007)). Under price dynamics similar to Bertsimas and Lo (1998) and a price impact function with permanent and transitory components, Almgren and Chriss (2000) derive an efficient frontier of optimal trading strategies that explicitly trade off execution costs with the risk of adverse price movements when traders have mean-variance utility. Linear price impact functions allow for closedform solutions. The expected cost-minimizing strategy of Bertsimas and Lo (1998) is optimal for risk-neutral agents. Risk averse agents will generally liquidate the portfolio more rapidly in order to reduce the variance of the execution cost. Thus, these papers imply a trading trajectory that executes quickly at the beginning of the trading horizon and slows down as time passes. Hora (2006) uses a different preference specification that leads to an optimal trading strategy that trades rapidly at the beginning and end of the trading horizon and more slowly in the middle of the period. The horizon over which trading takes place is generally unspecified in the models and could be a day, month, year, or some other interval. Almgren and Lorenz (2006) argue that a trading horizon of one day is consistent with the manner in which many institutions trade. In line with this view, Breen, Hodrick, and Korajczyk (2002) find that $92.5 \%$ of a sample of institutional orders are completed the same day that trading is initiated. The use of algorithmic trading has grown considerably and, as of 2007, was estimated to account for one-third of equity trading volume in the United States (Hendershott, Jones, and Menkveld (2009)).

The autocorrelation of fund flows and the use of trading algorithms discussed above imply the existence of daily patterns in volume and order imbalances in individual stocks. If Fund A receives fund flows today and buys stock XYZ using the Almgren and Chriss (2000) algorithm, then A will trade XYZ more aggressively in the morning and less aggressively as the day progresses. Since fund flows are autocorrelated, Fund A is likely to receive fund flows tomorrow. Further, because Fund A is likely to buy XYZ tomorrow using the same trading algorithm, volume and order imbalances 
in XYZ are likely to arise tomorrow at the same time as today. Anecdotal evidence indicates that this periodicity may be likely to happen even without explicitly invoking trading algorithms. Large equity managers have demanding schedules that involve client meetings, staff meetings, compliance, and reporting. The decision to buy or sell stock may require research analysis or portfolio optimization. The manager must then decide whether to use brokers or whether to relay the transactions through a crossing network such as Posit, Island, or Archipelago. These networks cross at certain times of the day, leaving unfilled orders at the end of the crossing. A busy manager may therefore find it cheap and expedient to execute these orders at specific times of the day, leaving the remaining time available for other trading, research, and risk management activity. The use of a trading algorithm that specifies a particular pattern of trades would only strengthen this periodicity. Additionally, some types of institutional traders may have other reasons for demonstrating periodicity in trading. Index funds, for example, tend to trade at the close to reduce tracking error.

The above conjectures about trading behavior imply periodicity in volume and order imbalance. Indeed, there is extensive evidence of intraday patterns in volume and volatility (e.g., Wood, McInish, and Ord (1985), Harris (1986), Jain and Joh (1988), and Pagano, Peng, and Schwartz (2008)). However, while intraday patterns of volume and volatility can be justified with models of discretionary liquidity trading (e.g., Admati and Pfleiderer (1988) and Hora (2006)), predictable patterns in returns are harder to explain. We consider several possibilities, none of which fully explain the periodicity. For instance, a long-standing literature on intraday return patterns (e.g., Wood, McInish, and Ord (1985), Harris (1986), and Jain and Joh (1988)) shows that average returns tend to be higher at the beginning and end of the trading day. Our observed patterns are not consistent with a simple mean shift at the beginning and end of the day. Additionally, the literature documents a number of seasonal return patterns, say at the monthly or quarterly frequency, in stock returns. Some of this periodicity is consistent with predictable patterns of trading by investors. For example, Keim (1989) finds that turn-of-the-year trading patterns induce patterns in the probability that trades occur at the ask price versus the bid price. He finds that this trading pattern explains the size-related turn-of-the-year effect in stock prices. However, we find that the return continuation at daily intervals is not due to the effect found in Keim (1989), that is, to changes in the frequency of trades occurring at either the bid or ask prices. Our intraday return pattern is stronger over the first and last half-hours of the trading day, as one might expect given the patterns in volume and volatility, but remains statistically significant over the other periods 
of the day. Thus, the intraday return pattern is not due simply to uniformly high returns at the beginning and end of the trading day.

Markets may also respond to news that arrives on a daily schedule. Newscasts and conference calls occur at regular times of the day, and behavioral biases may cause traders to overreact or underreact to salient news (Tversky and Kahneman (1974)), leading in turn to return periodicity. Our observed return periodicity is consistent with the information acquisition model of Holden and Subrahmanyam (2002) if agents tend to become informed at intervals that correspond to our observed periodicity.

Further, if there are regular news cycles, then traders may be reluctant to hold stocks during risky times. For example, if market-wide information is released at 3:30 PM, then high beta stocks may have a higher return premium at those times. This suggests that daily phenomena will be more pronounced among risky stocks than safe stocks. We do not find support for this hypothesis.

We also study whether the return periodicity is driven by similar patterns in order flow and liquidity. We show that trading volume has similar patterns to returns, that is, firms that experience a relatively high change in their trading volume over a particular half-hour interval typically experience a high change in their volume during the same half-hour interval during each of the next few days. Although related, the periodicity in trading volume does not completely explain the periodicity of returns. When we split volume into that due to large versus small trade size, both measures of volume show daily periodicity, but neither explains the daily return periodicity. Oddly, the level of order imbalance (OI) does not exhibit obvious periodicity, even when partitioned into small versus large trades. However, changes in order imbalance exhibit periodicity similar to returns, but the pattern in order imbalances does not explain the pattern in returns. Bid-ask spreads exhibit similar intraday periodicity as that of returns, but spreads do not explain the return patterns.

Several other tests suggest that the intraday periodicity at the daily frequency is not simply an artifact of previously shown patterns. For example, it is not concentrated in any particular weekday, and therefore is not a manifestation of the day-of-the-week effect (see French (1980)), and it is not concentrated in any particular month (see Heston and Sadka (2008a, 2008b)). The effect is also not particularly related to the turn-of-the-month effect (Ariel (1987)) or the turn-of-the-quarter effect (Carhart et al. (2002)). The pattern of intraday returns is highly persistent, lasting for two months (40 trading days). It is not due to a particular market capitalization group, inclusion in the S\&P 500 index, nor is it a manifestation of intraday movements in systematic risk. There is substantial 
evidence of intraday periodicity of return volatility (Andersen and Bollerslev (1997)), which we also find in our sample. However, patterns in volatility do not explain our return periodicity.

Finally, we compare the results for the post-decimalization period to prior periods, which include 1993 to 1997, for which the minimum price increment was one-eighth of a dollar, and 1997 to 2000, for which the minimum price increment was one-sixteenth of a dollar. We find that the statistical significance of the intraday periodicity is greatest in the post-decimalization period.

In Section I we present our main findings regarding return continuation at daily frequencies and short-term return reversals. In Section II we study possible cross-sectional explanations for the findings. In Section III we study the properties of volume, order imbalance, volatility, and liquidity while we provide additional diagnostics in Section IV. Our conclusions are presented in Section V.

\section{Patterns of Resilience in Intraday Stock Returns}

We begin this study by measuring intraday persistence in the cross-section of stock returns. It is well known that short-term stock returns are negatively autocorrelated (e.g., Lehmann (1990) and Lo and MacKinlay (1990)). While this phenomenon does not occur in the model of Glosten and Milgrom (1985), in which the spreads are due solely to adverse selection caused by informed traders, it appears in other models with bid-ask spreads (Roll (1984) and Glosten and Harris (1988)), specialist inventory effects (Stoll (1978)), or other non-adverse selection costs associated with market-making. Here we study the resilience of stock prices based on the pattern of autocorrelation over various horizons.

\section{A. Methodology and Main Results}

Our sample of firms consists of all New York Stock Exchange (NYSE) listed firms from January 2001 through December 2005 that we are able to match with the NYSE Trade and Quotation (TAQ) database. We retain only assets whose CRSP share codes are 10 or 11, that is, we discard certificates, ADRs, shares of beneficial interest, units, companies incorporated outside the U.S., Americus Trust components, closed-end funds, preferred stocks, and REITs. The matching yielded data for 1,715 firms. The period of study is chosen to substantially overlap the period of decimalization, the transition to which occurred between August 2000 and January 2001. We use the TAQ database to calculate intraday stock returns. For each stock we calculate returns over halfhour intervals. This gives 13 intraday intervals per trading day from 9:30 a.m. to 4:00 p.m. This excludes after-hours trading and overnight open-close price movements. Note that settlement on stock transactions occurs after the end of the trading day. This means that trades at different times 
do not need to earn the risk-free rate intraday. In other words, intraday stock returns compensate for liquidity and risk, not for the time value of money. In addition to returns, we also measure changes in volume defined as the change in the logarithm of the number of shares traded over a half-hour interval. The return and volume data provide measures of price and quantity movements of individual stocks throughout the day.

We analyze intraday stock returns using the cross-sectional regression methodology of Jegadeesh (1990). For each lag, $k$, we run cross-sectional regressions of half-hour stock returns on returns lagged by $k$ half-hour periods,

$$
r_{i, t}=\alpha_{k, t}+\gamma_{k, t} r_{i, t-k}+u_{i, t}
$$

where $r_{i, t}$ is the return on stock $i$ in the half-hour interval $t$. The slope coefficients $\gamma_{k, t}$ represent the response of returns at half-hour $t$ to returns over a previous interval lagged by $k$ half-hour periods, the "return responses." ${ }^{1}$ In addition to the simple regression (1), we also use a multiple regression to estimate all return responses up to five trading days jointly

$$
r_{i, t}=\alpha_{t}+\gamma_{1, t} r_{i, t-1}+\gamma_{2, t} r_{i, t-2}+\ldots+\gamma_{65, t} r_{i, t-65}+u_{i, t} .
$$

Both the simple regression and the multiple regression use all firms with returns available in intervals $t$ and $t-k$. Initially we present unconditional return responses, averaging over different times of the day $t$ for each lag $k$. Later we examine conditional averages, including time-of-day variation in the magnitude of these effects. We calculate the pattern of return effects by averaging return responses over time for half-hour lags $k$. Note that using cross-sectional regression in this way is different from measuring the autocorrelation of stock returns. In particular, the cross-sectional regression subtracts an overall market effect, which reduces variance and focuses on returns relative to other stocks.

Figure 1 presents the average return responses across different lags (Panel A) for lags up to one week. ${ }^{2}$ The data are scaled so the units for $\widehat{\gamma}_{k}$ are basis points. With 13 half-hour intervals per day and five trading days per week, this produces 65 lagged intervals. Consistent with the previous literature, the first several return responses are negative. This means that stock returns experience a reversal period lasting several hours. These reversals may be a manifestation of bid-ask bounce, time variation in the frequency of trades occurring at bid versus ask prices (as in Keim (1989)), or temporary liquidity dislocations that take some time for liquidity providers to accommodate. We disentangle these effects.

[Figure 1 here] 
The most striking pattern in Figure 1 is that, following the reversal period, the return responses are positive, peaking at horizons that are exact multiples of 13 half-hours, or one trading day. The figure shows clear periodicity at daily intervals. Panel B of Figure 1 plots the $t$-statistics of $\widehat{\gamma}_{k}$, as estimated in Fama and MacBeth (1973). The $t$-statistics show the same type of periodicity. Over the first week the smallest $t$-statistic at the daily frequency (lags $13,26,39,52$, and 65 ) is 9.62 .

Table I shows that the simple regression return responses are highly statistically significant at almost all lags. The Internet Appendix (Table IA.I) ${ }^{3}$ shows the results of the multiple regression responses are similar to the simple regression in magnitude, generally with greater statistical significance. Over the period of one calendar day these results indicate that returns are temporarily reversed but then rebound.

[Table I here]

Looking beyond 13 lags, the return effects over half-hour intervals on subsequent days remain largely negative, with statistically significant positive effects at multiples of 13 lags, that is, 26, 39, 52 , and 65 . It appears that temporary price pressure is reversed at virtually all future times except at the same time interval on subsequent days.

Since the simple regression produces results almost identical to the multiple regression, the univariate specification (11) seems adequate. By focusing on one lag at a time, we can easily examine a number of different lags.

The implicit portfolio weights in the portfolio returns obtained from the estimated regression coefficients, $\widehat{\gamma}_{k}$, could be rather extreme since, as mentioned above, $\widehat{\gamma}_{k}$ is the return on a zero-cost portfolio whose excess return $k$ periods ago is $100 \%$ (Lehmann and Modest (2005)). Therefore, we also study the returns to relatively well-diversified (equal-weighted) long-short portfolios formed on the basis of lagged half-hour returns. Using the methodology of Jegadeesh and Titman (1993), we sort stocks into equal-weighted deciles based on their returns over a previous half-hour interval. Figure 2 and Table II present these results extending the lags to roughly two trading months (520 lags). We form portfolios of "losers" and "winners" and calculate equal-weighted returns (in basis points) on portfolios of stocks that had the lowest and highest $10 \%$ of returns $k$ periods ago, respectively. The column labeled "10-1" is the average return on a portfolio that is long the winners portfolio and short the losers portfolio. ${ }^{4}$ This long-short average return is plotted as a function of $k$ in Figure 2, along with the associated $t$-statistics.

[Figure 2 and Table II here] 
Based on a half-hour return on a given day, the average difference between the top decile of winners and the bottom decile of losers is 3.01 basis points at the same time on the next day. To get a sense of the economic magnitude of this periodicity, we consider a few comparisons. Being able to capture three basis points is similar in magnitude to the daily equity risk premium. ${ }^{5}$ The effect may be larger than the equity risk premium in terms of incremental Sharpe ratio since one needs to bear one day of risk to earn the risk premium but only needs to shift trade a fraction of a day to take advantage of the periodicity. Being able to capture three basis points is also similar in magnitude to saving a $\$ 0.01$ commission on a stock priced at $\$ 30 /$ share (which is in line with institutional commission rates on common stock). Alternatively, Gurliacci, Jeria, and Sofianos (2009) find that the typical quoted half-spread of S\&P 500 stocks in their benchmark ("normal" volatility) period (May 2008) is 1.7 basis points, which means that the average return predictability is of the same order of magnitude as twice the quoted half-spread (the return difference requires two trades, so the relevant comparison is twice the half spread). ${ }^{6,7}$ Jeria and Sofianos (2008) evaluate institutional order executions for two alternative algorithms and find that there is a 2.3 basis point difference between the implementation shortfall for the average order. They use this result to recommend that traders migrate from one algorithm to the other. Thus, for high-frequency institutional traders, the effect appears to be economically consequential, particularly for high frequency traders ("Rise of the Machines," The Economist, July 30, 2009).

The average return difference remains positive, albeit smaller, on subsequent days. The difference remains positive and statistically significant for up to 40 days (520 half-hours). It appears there is a persistent and predictable pattern in intraday stock transaction prices. When a stock goes up on one day, buyers earn a return premium by buying the stock prior to (or delaying sales until after) the same time interval on future days. Conversely, sellers provide a discount by selling at this time when they could expect a higher average price 30 minutes later.

\section{B. Reversals: Resiliency, Bid-Ask Bounce, and Periodicity in Order Imbalances}

A market is resilient when it replenishes depth rapidly after having been depleted by large transactions (see, for example, Coppejans, Domowitz, and Madhavan (2004) and Large (2007)). We expect to see large price movements due to these types of trades, followed by rapid reversals as new limit orders replenish the order book. In less resilient markets, the reversal will take longer, leading to a longer period of return reversals. Therefore, the observed negative values of $\widehat{\gamma}_{k}$ at low values of $k$ (Figure 1, Table I, and the Internet Appendix Table IA.I), and the negative returns on the winners minus losers portfolios (Figure 2) may be due to liquidity shocks when the market is 
not very resilient. While this cause may be plausible for the negative $\widehat{\gamma}_{k}$ for $k$ between one and eight, it is unlikely to explain the negative $\widehat{\gamma}_{k}$ for lags corresponding to two to five days ago.

An alternative explanation for the negative values of $\widehat{\gamma}_{k}$ is some combination of bid-ask bounce and time variation in the frequency of trades occurring at bid versus ask prices (as in Keim (1989)). In Roll (1984) there is an equal chance that a trade occurs at the bid price or the ask price. This implies that order flow is not autocorrelated, but that bid-ask bounce induces negative first-order autocorrelation in returns. In the Roll model there is no autocorrelation in returns at lags greater than $k=1$. However, the empirical evidence shows that order imbalances (signed order flow) tend to be autocorrelated (Sadka (2006)), so trades at the ask will tend to be followed by trades with a higher probability of being at the ask. This type of pattern could lead to reversals that are not immediate (that is, return autocorrelation beyond the first lag).

Of course, both liquidity imbalances and bid-ask bounce are likely to be present in the data. To distinguish between lack of resiliency and bid-ask bounce, we reestimate $\widehat{\gamma}_{k}$ using returns calculated from only bid prices (defined as the current bid quote for the transactions), from only ask prices, and from only midpoint prices, instead of from transaction prices. If the observed return reversal pattern is due to lack of resiliency, we should see return reversals using the bid-to-bid, ask-to-ask, and midpoint-to-midpoint returns. Conversely, if the reversals are merely due to bid-ask bounce, then there should be no return reversals using bid-to-bid or ask-to-ask returns.

In Figure 3 we plot the estimates, $\widehat{\gamma}_{k}$, and their $t$-statistics using bid-to-bid, ask-to-ask, and midpoint-to-midpoint returns. The only reversal that is statistically significant is that for $k=1$. This is consistent with the market taking longer than 30 minutes, but less that 60 minutes, to restore shocks to liquidity, consistent with the evidence in Chordia, Roll, and Subrahmanyam (2005). Thus, there is evidence of a resiliency effect at lag $k=1$ only, and all of the reversals evident using transaction returns beyond lag $k=1$ disappear using bid-to-bid and ask-to-ask returns. Our inference is that the negative values of $\widehat{\gamma}_{k}$ beyond lag 1 shown in Figures 1 and 2 are due to bid-ask bounce. Once that effect is removed, there seems to be some evidence of momentum (positive values of $\widehat{\gamma}_{k}$ ) in returns using bid-to-bid and ask-to-ask prices.

[Figure 3 here]

While the short-term reversals evident in Figures 1 and 2 are consistent with microstructure theory and give us a coherent picture of resiliency and bid-ask bounce, the positive return responses at daily frequencies are much more difficult to explain. We now turn to the nature of these return 
continuations and their relation to other periodicity anomalies. We first study patterns across past-return deciles and across different times of the day.

\section{Patterns Across Past-Return Deciles}

We develop and compare strategies that hold stocks over a single half hour interval to focus on the periodic return pattern based on past returns at different lags. Table III shows the performance of stock deciles ranked on their performance in previous half-hour intervals. The strategies labeled "daily" sort stocks based on one half-hour interval at the same time on a previous day $(k=$ $13,26,39,52$, or 65$)$. The top row lists the decile number, with decile $j$ at time $t$ being made up of those stocks whose returns are in the $j^{\text {th }}$ decile (from lowest to highest) in period $t-k$ for $k=13,26,39,52$, or 65 .

\section{[Table III here]}

The nondaily strategies rank assets into deciles based on average returns over a previous 24hour period except the daily lags. For example, the Day 1-Nondaily strategy forms portfolios on the basis of their average returns over lags 1 through 12, the Day 2-Nondaily strategy uses average returns over lags 14 through 25, etc. By studying the returns on these decile portfolios we can observe any nonlinearity in the relation between average returns across deciles, such as whether the daily pattern is concentrated in the upper or lower performing deciles.

The table shows most of the statistical and economic significance comes from the extreme deciles. For example, the worst nondaily losers over the previous day earn an average of 3.16 basis points, while the best nondaily winners lose 1.51 basis points per half-hour holding period. The average returns are nearly monotonic across intermediate deciles. The signs are reversed for the daily decile strategies. For example, the worst decile of losers over the same interval of the previous day continue to lose an average of 1.35 basis points, while the best decile of daily winners earn 1.66 basis points per half-hour interval. The intermediate decile average returns are monotonic, but most of the significance comes from the lowest and highest deciles.

The magnitude of the daily response is most substantial on the first day, however there is a smaller persistent effect. For lags beyond one day, the average daily winners minus losers decile spreads remain above one basis point per half-hour for lags of up to five days (with $t$-statistics above 8.7). By contrast, the magnitude of the negative nondaily winners minus losers decile spreads is less than one basis point. Average decile spreads for nondaily strategies are statistically significant for lags of at least four days. 


\section{Time of Day}

The return patterns in Figures 1 and 2 reflect average behavior throughout the day. Investors can pay up to three extra basis points, on average, to execute trades at a particular time of day. However, it is possible this effect is concentrated at certain times of the day. We first investigate whether the intraday pattern is an artifact of biases in opening or closing prices. Overnight orders are executed at the open, and many traders (for example, index funds concerned with minimizing daily tracking error) place market-on-close orders. Temporary price distortions caused by opening and closing procedures might produce predictability in stock returns that does not affect stock prices at other times of the day.

Table IV shows the excess return of winners minus losers decile spread strategies during different half-hour intervals throughout the day. Since the nondaily effect is mainly driven by bid-ask bounce, we only report the results for the daily strategy. The full results are reported in the Internet Appendix (Table IA.III). For Day 1, the daily decile spreads are sorted based on the returns for lag 13, while the nondaily spreads (in the Internet Appendix) are sorted based on the average returns for lags one through 12. The return effect is quite pronounced in the first and last half-hours of trading. The Day 1 daily decile spread earns over 11 basis points in the opening half-hour, while the Day 1 nondaily strategy loses over eight basis points. This is a difference of 19 basis points between these strategies in the opening half-hour. Similarly, the Day 1 daily decile spread earns over eight basis points near the close of trading, while the corresponding nondaily strategy loses 11 basis points. A smaller effect remains during the middle of the day. The Day 1 daily strategy earns positive average excess returns in every half-hour interval from 10:00 a.m. to 3:30 p.m., averaging 1.75 basis points over this period. Meanwhile, the Day 1 nondaily strategy declines in nearly every half-hour period, losing 3.74 basis points per half-hour over this period. This is a consistent pattern throughout the day. At longer lags, labeled Day 2 through Day 5 in Table IV, the return patterns are of a similar shape but smaller in absolute value. When we average the second through twelfth intervals (that is, average returns from 10:00 a.m. to 3:30 p.m.), all the daily spreads are significantly different from zero at conventional levels while the nondaily spreads are statistically significant for Day 1 and Day 2.

\section{[Table IV here]}

Figure 4 shows that the periodic daily pattern of return strategies has a similar shape but different magnitudes at different times of the day. The daily strategies earn a premium in the first 
half-hour of trading, the last half-hour, and in the intermediate intervals from 10:00 to 3:30. This evidence indicates that while the periodicity at daily intervals is stronger at the beginning and end of the trading day (as can be seen from the magnitudes of the coefficients in the left side of the figure), it exists at all times (as can be seen from the magnitudes of the $t$-statistics in the right side of the figure) ${ }^{8}$ For the intermediate intervals there is more anticipation of the periodicity at lag 13 since the coefficients are significantly positive for lags 10 through 13 . The top and bottom graphs in Figure 4 show some leakage from the closing half-hour to the next day's opening half-hour. Figure 4 is consistent with the argument that the patterns are mainly, although not exclusively, driven by trading at or near the opening or closing of the trading day.

[Figure 4 here]

\section{Potential Cross-sectional Explanations}

The pattern of daily return continuation has several potential explanations. One concern is the effect of data errors caused by thinly traded stocks. After addressing this, we consider whether size or risk can explain the return pattern. We also examine whether trading profits of strategies based on return periodicity are wiped out by paying the bid-ask spread.

\section{A. Low Price and Thin Trading}

A major concern is whether the return continuation is driven by thinly traded stocks with inaccurately or irregularly measured returns. Returns on low-priced stocks are most strongly affected by discreteness. However, since our sample is from the post-decimalization period, we do not expect discreteness to be a major cause of return periodicity.

Many stocks may not be traded throughout a half-hour interval. For example, a stock might trade once at 12:01, and then a second time at 12:02, without any further activity before 12:30. While it is not clear whether this would impart a daily pattern in measured returns, we verify whether our results are contaminated by such inaccurate or illiquid returns.

Table V addresses these issues by repeating the decile spread analysis of Table III while eliminating low-priced and thinly traded stocks. We define low-priced stocks as stocks with share prices below $\$ 5$. Removing the low-priced stocks decreases the nondaily reversal effect (reported in Internet Appendix Table IA.IV). The average nondaily decile spread on Day 1 changes from -4.67 basis points per half-hour in Table III to -1.77 basis points (with a $t$-statistic of -8.07) in the Internet Appendix (Table IA.IV). Given that the reversals beyond lag $k=1$ are driven by bid-ask bounce, one would expect some attenuation since low-priced and thinly traded stock tend to have higher 
percentage spreads.

[Table V here]

The daily continuation effect shrinks less than the nondaily reversal effect. Table V shows that the daily decile spread earns 1.93 basis points (with a $t$-statistic of 10.34) on Day 1, whereas it earns roughly three basis points with all stocks in Table III. In both Table III and Table V the daily decile spread remains roughly one basis point per half-hour on Days 2 through 5 .

To address problems of thin trading, we also eliminate stocks that do not have an average of at least 10 trades per half-hour interval over the previous month. In other words, we use stocks that average at least one trade every three minutes. This retains roughly $80 \%$ of the stocks in our sample. The second column of Table $\mathrm{V}$ shows that this restriction does not affect the results as much as eliminating the low-priced stocks. The daily decile spread earns 2.45 basis points (with a $t$ statistic of 18.44) per half-hour on Day 1, while the nondaily decile spread (in the Internet Appendix (Table IA.IV)) loses 3.17 basis points (with a $t$-statistic of -19.44). Results on subsequent days are similar to returns using the full sample, reported in Table III. Overall, the reversal and continuation patterns are not an artifact of mismeasurement of the return on low-priced stocks or mistiming of returns on thinly traded stocks.

\section{B. Size and Transactions Costs}

A concern about the nature of intraday patterns in stock returns is market depth. If there is a return premium at certain times of the day then it may be compensation for illiquidity that makes stocks difficult to trade at efficient prices during those times. For example, Admati and Pfleiderer (1988) develop a model where traders pool trades in certain periods of the day in order to take advantage of the increased liquidity of pooling. This implies a pattern primarily among smaller and less liquid stocks that face larger adverse selection problems. To the extent that firm size proxies for variation in adverse selection problems, it provides a control variable.

In Table VI we sort stocks into three groups based on market capitalization at the end of the previous calendar year. In particular, the table reports the decile spread strategies separately for small-, medium-, and large-capitalization firms, where each group contains $33 \%$ of the sample. The nondaily strategies on Day 1 (reported in the Internet Appendix (Table IA.V)) have returns that are more negative among small firms. This is consistent with small-cap firms having larger proportional spreads. The Day 1 nondaily decile spread loses more than 10 basis points in the opening half-hour and more than 22 basis points in the closing half-hour, while averaging a loss of 
more than eight basis points in the mid-day half-hour intervals. Conversely, the daily decile spread strategies are more profitable with small stocks. The Day 1 daily strategy averages more than five basis points per half-hour. In contrast, average returns are in the range of one to three basis points for medium and large stocks. The average excess returns for strategies based on longer daily and nondaily lags are smaller and do not differ much across size categories. However, almost all daily strategies maintain statistical significance at the $95 \%$ level in all size categories at the open, mid-day, and close. This indicates that while a liquidity/microstructure effect explanation may have merit, return periodicity is not associated exclusively with small firms.

\section{[Table VI here]}

An important consideration is the magnitude of transaction costs associated with the trading strategies implicit in our reported returns. We find predictable excess returns of several basis points within a half-hour interval based on transaction prices. However, a trader with no other motive for trade must pay the ask price or accept the offer price to get immediate execution and avert time slippage. Larger orders also lead to larger price impacts. Table VII reports the decile spread results for strategies that buy at the offer and sell at the bid. The average results are negative for all size categories at all times of the day, indicating that the periodicity we find does not indicate a pure profit opportunity. It is important to remember that these results involve the round-trip transaction costs of two different long-short decile strategies. Therefore, they incur the average cost of four transactions. For example, among small stocks the average decile spread return on the Day 1 Nondaily strategy (reported in the Internet Appendix (Table IA.VI)) is -25.03 basis points, and the average decile spread return on the Day 1 Daily strategy is -23.78 basis points. The Internet Appendix (Table IA.V) shows that the Day 1 Nondaily strategy lost 9.92 basis points among small stocks while Table VI shows that the Day 1 Daily strategy gained 5.16 basis points. Comparing the pre-transaction cost returns in Table VI to the post-spread return in Table VII, we see that the implied one-way spread cost is between four and seven basis points. While the difference between the performance of Daily and Nondaily strategies does not cover the costs of two round-trips, it often compares favorably with the magnitude of one-way transaction costs. This suggests that many investors have a demand for immediate execution of trades and are not willing to shift their trades by 30 minutes to take advantage of the periodicity.

[Table VII here] 
These trading strategies are not likely to be profitable for stocks with large bid-ask spreads. Therefore, the results in Table VII exclude stocks that have a quoted relative spread of more than 10 basis points at the beginning of a given trading interval. We also perform the analysis restricted to stocks with spreads less than five and 25 basis points, respectively. The results are similar to those in Table VII. Note that very few small stocks have spreads less than five basis points, so there is no point conditioning on less than five basis points. Since our reported raw profits are not greater than 25 basis points, we do not condition on spreads greater than 25 basis points. The results suggest that it is quite difficult to profit from these type of intraday strategies without some exogenous desire to trade.

The transaction costs for medium and large stocks are substantially smaller than those for small stocks. Table VII shows that for medium stocks the average decile spread results are roughly -20 basis points and for large stocks they are roughly -14 basis points. This corresponds to one-way trading costs of less than five basis points. The losses of the Nondaily and Daily strategies in Table VII and the Internet Appendix (Table IA.VI) are smaller for medium and large stocks than for small stocks.

Our evidence for the nondaily reversal effect is consistent with the results of Avramov, Chordia, and Goyal (2006), who find that the reversals at weekly and monthly intervals are smaller than transactions costs.

\section{Beta}

If high frequency changes in risk or liquidity influence investors' demand for assets, then we might find periodicity in returns due to periodicity in risk or liquidity. For example, stocks might be riskier at certain times of the day when news is released, or they might be subject to institutional transactions that follow a daily cycle. This section explores these possibilities.

A priori, it seems unlikely for stocks to have large fluctuations in systematic risks during the day because companies do not change their financial exposures from hour to hour. On the other hand, some economic series are released at scheduled times, and firms may have exposure to systematic news released at those times. In this case traders may be reluctant to hold stocks at these risky times. To assess this possibility we control for risk by regressing stock returns on the equalweighted market index. To correct for nonsynchronous trading (Dimson (1979)), we include the contemporaneous half-hour market return along with 13 leads and lags. The Internet Appendix (Table IA.VII) reports the average intercepts from these regressions. Since the intraday interest rate is effectively zero, these intercepts have the interpretation of risk-adjusted returns. 
The results reported in the Internet Appendix (Table IA.VII) resemble the previous results using unadjusted average returns. The average risk-adjusted return on decile return spreads of lag 13 winners in excess of lag 13 losers is 3.03 basis points. The decile spread has a risk-adjusted return of -4.65 basis points when portfolios are formed on the basis of returns over lags 1 through 12. These effects are particularly pronounced in the first half-hour and last half-hour of the day, but remain statistically significant in the middle of the day.

The average risk-adjusted returns for the daily decile spreads continue to be substantial in the opening and closing half-hour even when sorting on half-hour returns up to five business days ago. The Day 5 average decile spread for $k=65$ is 4.84 basis points in the first half-hour, and 3.42 basis points in the last half-hour. The effect is much smaller in the middle of the day, less than one basis point, but remains statistically significant. Therefore, controlling for market risk does not eliminate the daily pattern.

These results also show that the periodicity is not an artifact of all stocks having high returns at certain times of the day and low returns at others, that is, simple mean shifts across half-hour intervals. If this were driving the results, risk-adjusted returns should eliminate the effect since the risk adjustment takes out the market return. However, the risk-adjusted returns in the Internet Appendix (Table IA.VII) are almost identical to the unadjusted returns in Table IV.

\section{Volume, Order Imbalance, and Related Variables}

Autocorrelated fund flows and a preference for trades at particular times of the day should lead to periodicity in quantity variables (e.g., volume) and signed quantities (e.g., order imbalance), and possibly also in volatility and liquidity. As discussed above, at the aggregate level there is an extensive literature on intraday patterns in volume and volatility. This literature is consistent with periodicity in volume, order imbalance, volatility, and liquidity of the type observed in returns. However, there may be different intraday patterns that are also consistent with this literature (for example, an intraday step function in which the means of all variables are shifted each period). We wish to determine whether these variables have periodicity similar to returns. If they do, we would like to know if past observations of these variables explain the return periodicity. We find common periodic daily patterns in some of these variables, but show that these variables do not subsume the pattern of return predictability.

All these variables are quite persistent in their levels, so the response coefficients we observe by estimating equation (11) look different than those for returns in Figure 1. This is to be expected, 
given what we know about volume (Lo and Wang (2006)), order imbalance (Sadka (2006)), volatility (Bollerslev (1986)), and liquidity (Korajczyk and Sadka (2008)). In particular, the coefficients using levels of these variables are positive and decay very slowly. However, when we look at changes (order imbalance) and percentage changes (volume, volatility, and spreads) in these variables, many display patterns that look similar to the return patterns shown in Figure 1.

\section{A. Volume and Order Imbalance}

To address trading-demand explanations of periodicity, we repeat the cross-sectional regression using volume data,

$$
v_{i, t}=a_{k, t}+g_{k, t} v_{i, t-k}+u_{i, t}
$$

where $v_{i, t}$ is the percentage change in the volume of stock $i$ traded over the half-hour interval $t$. The means and $t$-statistics ${ }^{9}$ of the time series of volume responses are plotted in Panel A of Figure 5. The pattern for $\widehat{g}_{t}$ strongly resembles the return response pattern, $\widehat{\gamma}_{t}$, in Figure 1. In particular, the cross-sectional volume response effects are uniformly negative at all lags except multiples of 13. Figure 5 shows the pattern for 65 lagged half-hour intervals corresponding to one week of calendar lags. Like the pattern of return responses, the effect of volume responses lasts much longer. The Internet Appendix (Figure IA.1) shows that the strength of daily volume responses decays with longer lags, but remains positive and statistically significant for up to 520 half-hour lags, corresponding to 40 days. Together Figures 1 and 5 (and the longer-term results in Figure 2 and the Internet Appendix) show that the intraday cross-sections of daily return and volume display similar persistence lasting at least two months. Note that to the extent that volume and volatility are correlated, the volume pattern is consistent with the patterns in intraday volatility documented in Andersen and Bollerslev (1997).

\section{[Figure 5 here]}

It is probable that orders of different size convey different information, and have different execution costs. Small trades are primarily generated by individual investors, whereas large trades are preponderantly institutional (Campbell, Ramadorai, and Schwartz (2009)). To check for differential effects of volume generated by orders of different sizes, for each 30-minute interval we determine the volume generated by trades of less than 1,000 shares each (small trade volume) and the volume generated by trades of greater than or equal to 1,000 shares each (large trade volume). The Internet Appendix (Figure IA.2) shows the volume responses separately for small trades (Panel A) and large trades (Panel B). Percentage changes in both small- and large-trade volume exhibit 
the same pattern of periodicity that we see in returns in Figure 1 and in overall volume in Figure 5.

It is possible that institutions disguise large trades by breaking them into smaller ones, making it difficult to detect their trades. Regardless of our ability to identify specific types of trades, price pressure is presumably caused by one-sided volume, not by balanced trading. Therefore, we also look at the behavior of changes in signed volume, order imbalance. Order imbalance is defined as the net signed volume over the interval, where the sign is determined by a variant of the Lee and Ready (1991) trade classification algorithm. ${ }^{10}$ For example, if a time interval had 100,000 shares transacted that were classified as buyer-initiated and 75,000 shares transacted that were classified as seller-initiated, then the order imbalance for the period would be 25,000 shares (equal to 100,000 - 75,000). Panel D of Figure 5 shows that changes in order imbalance exhibit periodicity similar to but less pronounced than the periodicity in returns and volume.

The Internet Appendix (Figure IA.2) indicates that order imbalance does not exhibit any additional periodicity when partitioned into small versus large trades, in contrast to the momentum results of Hvidkjaer (2008). Indeed, the periodic pattern is absent in the order imbalance for large trades. Perhaps trade classification algorithms for identifying buyer-initiated versus seller-initiated trades produce noisy estimates for individual stocks over short horizons such as the half-hour intervals used here.

\section{B. Volatility and Bid-Ask Spreads}

Volatility tends to be high at the beginning and end of the trading day. In addition, Andersen and Bollerslev (1997) find high returns for the S\&P 500 composite stock index futures contract at the beginning and end of the day. It might be the case that movements in volatility are driving the return periodicity we observe here.

Movements in bid-ask spreads might also be related to movements in volume, volatility, and returns. For example, Admati and Pfleiderer (1988) derive a model in which traders who have discretion on when to trade prefer to coordinate trading at the same time. In this model, movements in volume, volatility, and liquidity are related, in equilibrium.

Figure 5 (Panel B) shows that percentage changes in volatility (measured by the absolute value of returns) exhibit intraday periodicity similar to that found for returns and volume.

If market activity in individual stocks has daily periodicity, then bid-ask spreads might follow the same pattern. We examine this to distinguish changes in execution costs from changes in underlying returns. In addition, since the high frequency return bias discussed in Blume and 
Stambaugh (1983) is related to bid-ask bounce, we wish to test whether the return patterns we find are related to systematic changes in bid-ask spreads. Panel C of Figure 5 shows the $t$-statistics from cross-sectional regressions in which percentage changes in spreads are regressed on their lagged values. Spreads do not exhibit the type of periodicity that we observe in returns. Therefore, it appears that the return periodicity is not merely an artifact of a spread-induced bias in estimated returns.

\section{Does Periodicity in Related Variables Explain Periodicity in Returns?}

We have shown that volume, order imbalance, and volatility have cross-sectional predictability with the same daily pattern as returns, whereas spreads do not. We now turn to the question of whether the periodicity in these variables explain the return predictability we observe. For example, daily patterns in institutional volume might affect stock liquidity and volatility at the same time each day. News cycles might trigger volume that causes investors to demand return premiums on a daily cycle.

To control for these additional variables, we run cross-sectional regressions of returns on lagged returns, including these additional lagged regressors. The regression is

$$
r_{i, t}=\alpha_{k, t}+\gamma_{k, t} r_{i, t-k}+\delta_{k, t}^{\prime} V_{i, t-k}+e_{i, t}
$$

where the vector $V_{i, t-k}$ includes percentage changes in volume (total shares traded during the lagged half-hour interval), volatility (absolute return), and relative bid-ask spread; and absolute changes in order imbalance. If return predictability is caused by predictable market activity based on these variables, then including them should diminish or even subsume the predictability of returns based on past returns. The values of $\widehat{\gamma}_{k}$ and the associated $t$-statistics are reported in Figure 6 .

[Figure 6 here]

Surprisingly, Figure 6 shows that the daily pattern of cross-sectional return predictability is unaffected by including the additional regressors. In other words, volume, order imbalance, and volatility have similar patterns of intraday predictability, but these variables do not explain daily return predictability.

As an additional refinement, we also regress returns on lagged returns, lagged small-trade volume, and lagged large-trade volume. The Internet Appendix (Figure IA.2) reports the results. The results show that the return periodicity is not subsumed by large- or small-volume either.

D. Pre-decimalization Results 
We focus our analysis in this paper on the post-decimalization period. In Table VIII we compare the return spreads on daily-frequency strategies (lags 13, 26, 39, 52, and 65) over our sample period to two additional sample periods corresponding to tick sizes of one-eighth and one-sixteenth of a dollar. We find that the statistical significance of the intraday periodicity is greatest in the postdecimalization period. This might be due to the increased use of trading algorithms (of the sort discussed above) by institutional investors. Results during the period when the tick size is onesixteenth are quite unreliable and, hence, we present additional results in Table VIII, Panel B, where the returns are Winsorized at the $1 \%$ level each month. That is, for each calendar month and each strategy (e.g., lag 13) we set all observations below (above) the $1^{\text {st }}\left(99^{\text {th }}\right)$ percentile to the value of the $1^{\text {st }}\left(99^{\text {th }}\right)$ percentile. The Winsorized results do not represent implementable trading strategies, but reduce the influence of outliers. The Winsorized results with one sixteenth tick size resemble the post-decimalization results, with slightly larger coefficients and slightly smaller $t$ statistics. The larger coefficients for the 1997 to 2000 period using the Winsorized estimator are not consistent with the hypothesis that the increase in algorithmic trading is driving the periodicity. The Winsorized returns over the 1993 to 1997 period, when the minimum price increment was one-eighth, have the smallest coefficients and $t$-statistics.

\section{[Table VIII here]}

\section{Additional Diagnostics}

The previous sections demonstrate a pattern of daily return continuation. This pattern is concentrated at the open and close, but also persists throughout the day and lasts for many days. Because of the novelty of this effect, it is important to verify that we have properly characterized the pattern (pervasive and not restricted to special days or periods). This section examines the pattern at a higher frequency and for different calendar periods.

\section{A. Five-Minute Interval Returns}

Figure 1 shows that when using half-hour intervals, sometimes there appears to be slight spillover from one time interval to adjacent intervals. While the return responses are most positive at exact multiples of one trading day (e.g., 13-half-hour lags), they are often slightly positive at adjacent intervals too (e.g., 12 or 14 half-hour lags). In this sense the choice of half-hour intervals seems adequate.

It is conceivable, however, that our choice of interval masks higher frequency phenomena. In addition, we would like to ensure that our results are not sensitive to the exact methodological choices. 
Therefore, we recalculate returns over five-minute intervals and estimate the return responses of equation (1). In this case there are 78 five-minute intervals during a trading day (omitting overnight returns).

Figure 7 shows these high-frequency results. Figure 7 looks like a noisy version of Figure 1. The negative return reversal is most pronounced for the first few five-minute lags, but persists for several days. And while there is a clear daily effect at daily multiples of 78 lags, the leakage of the neighboring five-minute intervals is also apparent. Overall, this graph confirms the previous results and indicates that the half-hour return intervals are fine enough to faithfully characterize high frequency returns.

[Figure 7 here]

\section{B. Day of Week}

A potential concern is the existence day-of-the-week effects. French (1980) finds that the stock market earns different average returns on different days of the week. In particular, average returns on the day following a weekend are lower than average returns on other days. Therefore, we check whether our daily effect is driven by a weekend effect or part of some other weekly pattern.

The Internet Appendix (Table IA.VIII) shows the performance of our daily and nondaily decile spread strategies on different days of the week. The effect is remarkably consistent throughout the week. The Day 1 nondaily strategy loses money at the open, mid-day, and close on every day. The amounts range from -3.51 basis points on Thursdays to -6.14 basis points on Mondays. Meanwhile the Day 1 daily strategy earns a positive premium when averaged over all half-hour intervals. This ranges from 2.62 basis points on Tuesdays to 3.44 basis points on Wednesdays. The results for longer lags are weaker, but they have the same sign and are usually statistically significant at the $5 \%$ level. We conclude that the daily results are not limited to a weekend effect or other weekday pattern.

\section{Calendar Month and Turn-of-Month}

There are well-known monthly patterns in the stock market. This includes both market-wide January effects and year-long seasonality (Rozeff and Kinney (1976), Bouman and Jacobsen (2002), and Kamstra, Kramer, and Levi (2003)) and cross-sectional return patterns such as the size effect at the turn of the year (Keim (1983)). In addition to ruling out weekday effects, we want to ensure that the daily pattern is not an artifact of some other monthly seasonal phenomenon.

The Internet Appendix (Table IA.IX) reports the decile spread strategies for every calendar 
month. Again, the results are strikingly consistent. The Day 1 nondaily strategy loses money in every calendar month, ranging from -2.88 basis points in June to -5.90 basis points in March. And the Day 1 daily strategy makes money in every month, ranging from 1.70 basis points in March to 7.80 basis points in November. The longer lag strategies have a similar pattern, albeit smaller and less consistent. Nevertheless, the Day 5 daily strategy is still profitable in every calendar month. The results are not limited to a particular time of year, and certainly not limited to the turn of the year.

While the results are not limited to turn of the year seasonality, they might be driven by intramonth patterns. Ariel (1987) shows that stocks earn a premium near the beginning and end of calendar months.

The Internet Appendix (Table IA.X) controls for turn-of-month effects by separately reporting the combined results for trading days that occur on the first or last day of the month. The results are remarkably consistent. The Day 1 and Day 2 nondaily strategies lose money at the open, mid-day, and close at both the turn of the month and the middle of the month, while the daily strategies make money at all times. With few exceptions the Day 3, 4, and 5 strategies maintain this pattern. The pattern is thus not related to turn-of-the-month effects.

\section{Index Membership}

If stocks rise and fall at the same time of day, then presumably there are buyers and sellers who persistently trade them at those times (with persistence in the direction of the trade). Index funds and benchmarked mutual funds are natural suspects for these actions. These funds may have large daily inflows or outflows and have an inelastic demand to invest those funds to replicate the index. To economize on trading activity they might perform "basket trades" at the open of trade, and to minimize tracking error they would have a motivation to trade near the close. This is consistent with previous results showing a strong effect at these times.

As a very rough cut at dealing with index fund behavior, we divide the sample between firms in the S\&P 500 and those that are not. The Internet Appendix (Table IA.XI) separates the decile spread results for S\&P 500 firms and non-S\&P 500 firms. The results for both daily and non-daily strategies are generally stronger among the non-S\&P 500 stocks. For example, the average decile spread based on the previous nondaily returns loses 5.58 basis points in the non-S\&P 500 stocks, but loses less than one basis point in the S\&P 500 index stocks. The Day 1 daily strategy earns 3.28 basis points with the non-S\&P 500 stocks, but earns only 2.19 basis points with the index stocks. This difference masks some interesting variation across the day. The closing-period daily 
effect is much larger, relative to the intraday effect, for the S\&P 500 stocks. For example, the ratio of the last period to intraday daily returns for Day 1 is $9.61(9.42 / 0.98)$ for S\&P 500 stocks and 4.94 for non-S\&P 500 stocks. The daily effect is larger for S\&P 500 stocks in absolute terms for Days 1 through 3, and in relative terms (versus the mid-day period) for all five days. For the opening half-hour the daily effects are smaller in absolute terms but larger in relative terms for $\mathrm{S} \& \mathrm{P}$ stocks. The large relative daily effect in the closing-period for S\&P 500 stocks is consistent with index fund trading at the close driving some of the daily effect. Similar effects are shown for large versus small firms (reported above in Table IV), so it is not clear whether this effect is an index effect or a large capitalization effect.

\section{Conclusion}

A growing literature suggests that institutional investment flows influence asset prices (e.g., Harris and Gruel (1986), Coval and Stafford (2007), Boyer (2008), He and Krishnamurthy (2008), Lou (2008), Vayanos and Woolley (2008)). We postulate that systematic trading and institutional fund flows lead to predictable patterns, not only in trading volume and order imbalances, but also in returns of common stocks. We study the periodicity of cross-sectional differences in returns using half-hour observation intervals in the period from January 2001 through December 2005. We document pronounced intraday return reversals due to bid-ask bounce, and these reversals last for several trading days. The market recovers from shocks to depth in less than 60 minutes. However, we find significant continuation of returns at intervals that are multiples of a day and this effect lasts for at least 40 trading days. This daily periodicity is of the same order of magnitude as current institutional commission rates and the quoted half-spread.

Changes in trading volume, order imbalances, and volatility exhibit similar patterns, but do not explain the return patterns. The return continuation at daily frequencies is more pronounced for the first and last half-hour periods. These effects are not driven by firm size, systematic risk premia, or inclusion in the S\&P 500 index (as a proxy for trading by index funds). The pattern is also not driven by particular months of the year, days of the week, or by turn-of-the-month effects. The periodicity at the daily frequency is observed when we also use bid-to-bid, ask-to-ask, or midpoint-to-midpoint returns, which implies that the periodicity is not merely due to patterns of where transactions prices occur relative to bid and ask prices.

The results are consistent with investors having a predictable demand for immediacy at certain times of the day. The pattern does not present a profit opportunity in the absence of other motives 
to trade, since strategies that attempt to take advantage of the daily periodicity lose money, after paying the bid-ask spread. However, the magnitude of the return pattern is sizeable relative to several components of transactions costs such as commissions and effective spreads. 


\section{References}

Admati, Anat, and Paul Pfleiderer, 1988, Theory of intra-day patterns: Volume and price variability, Review of Financial Studies 1, 3-40.

Almgren, Robert, and Neil Chriss, 2000, Optimal execution of portfolio transactions, Journal of Risk 3, 5-39.

Almgren, Robert, and Julian Lorenz, 2006, Bayesian adaptive trading with a daily cycle, Journal of Trading 1, 38-46.

Andersen, Torben G., and Tim Bollerslev, 1997, Intraday periodicity and volatility persistence in financial markets, Journal of Empirical Finance 4, 115-158.

Ariel, Robert A., 1987, A monthly effect in stock returns, Journal of Financial Economics 18, 161-174.

Avramov, Doron, Tarun Chordia, and Amit Goyal, 2006, Liquidity and autocorrelations in individual stock returns, Journal of Finance 61, 2365-2394.

Bertsimas, Dimitris, and Andrew W. Lo, 1998, Optimal control of execution costs, Journal of Financial Markets 1, 1-50.

Blackburn, Douglas W., William N. Goetzmann, and Andrey D. Ukhov, 2007, Risk aversion and clientele effects, Working paper, Indiana University.

Blume, Marshall E., and Robert F. Stambaugh, 1983, Biases in computed returns: An application to the size effect, Journal of Financial Economics 12, 387-404.

Bollerslev, Tim, 1986, Generalized autoregressive conditional heteroskedasticity, Journal of Econometrics 31, 307-327.

Bouman, Sven, and Ben Jacobsen, 2002, The Halloween indicator, 'Sell in May and go away': Another puzzle, American Economic Review 92, 1618-1635.

Boyer, Brian H., 2008, Comovement among stocks with similar book-to-market ratios, Working paper, Brigham Young University.

Breen, William J., Laurie Simon Hodrick, and Robert A. Korajczyk, 2002, Predicting equity liquidity, Management Science 48, 470-483.

Campbell, John Y., Tarun Ramadorai, and Allie Schwartz, 2009, Caught on tape: Institutional trading, stock returns, and earnings announcements, Journal of Financial Economics 92, $66-91$.

Carhart, Mark M., Ron Kaniel, David K. Musto, and Adam V. Reed, 2002, Leaning for the tape: Evidence of gaming behavior in equity mutual funds, Journal of Finance 58, 661-693.

Chordia, Tarun, Richard Roll, and Avanidhar Subrahmanyam, 2005, Evidence on the speed of convergence to market efficiency, Journal of Financial Economics 76, 271-292.

Coppejans, Mark, Ian Domowitz, and Ananth Madhavan, 2004, Resiliency in an automated auction, Working paper, ITG, Inc.

Coval, Joshua, and Erik Stafford, 2007, Asset fire sales (and purchases) in equity markets, Journal of Financial Economics 86, 479-512.

Del Guercio, Diane, and Paula A. Tkac, 2002, The determinants of the flow of funds of managed portfolios: Mutual funds vs. pension funds, Journal of Financial and Quantitative Analysis $37,523-557$.

Dimson, Elroy, 1979, Risk measurement when shares are subject to infrequent trading, Journal of Financial Economics 7, 197-226.

Engle, Robert, and Robert Ferstenberg, 2007, Execution risk: It's the same as investment risk, Journal of Trading 2, 10-20. 
Fama, Eugene F., 1976, Foundations of Finance (Basic Books, New York).

Fama Eugene F., and James D. MacBeth, 1973, Risk, return, and equilibrium: Empirical tests, Journal of Political Economy 81, 607-636.

Frazzini, Andrea, and Owen A. Lamont, 2008, Dumb money: Mutual fund flows and the crosssection of stock returns, Journal of Financial Economics 88, 299-322.

French, Kenneth R., 1980, Stock returns and the weekend effect, Journal of Financial Economics $8,55-69$.

Glosten, Lawrence R., and Lawrence E. Harris, 1988, Estimating the components of the bid/ask spread, Journal of Financial Economics 21, 123-142.

Glosten, Lawrence R., and Paul R. Milgrom, 1985, Bid, ask and transaction prices in a specialist market with heterogeneously informed traders, Journal of Financial Economics 14, 71-100.

Goyenko, Ruslan Y., Craig W. Holden, and Charles A. Trzcinka, 2009, Do liquidity measures measure liquidity? Journal of Financial Economics 92, 153-181.

Grinold, Richard C., and Ronald N. Kahn, 2000, Active Portfolio Management: A Quantitative Approach for Producing Superior Returns and Selecting Superior Returns and Controlling Risk, 2nd edition (McGraw-Hill, New York).

Gurliacci, Mark, David Jeria, and George Sofianos, 2009, When the going gets tough, the algos get going, Journal of Trading 4, 34-44.

Harris, Lawrence, 1986, A transaction data study of weekly and intradaily patterns in stock returns, Journal of Financial Economics 16, 99-117.

Harris, Lawrence, and Eitan Gurel, 1986, Price and volume effects associated with changes in the S\&P 500 list: New evidence for the existence of price pressures, Journal of Finance 41, $815-829$.

Hasbrouck, Joel, 2009, Trading costs and returns for U.S. equities: Estimating effective costs from daily data, Journal of Finance 64, 1445-1477.

He, Zhiguo, and Arvind Krishnamurthy, 2008, Intermediary asset pricing, Working paper, Northwestern University.

Hendershott, Terrence, Charles M. Jones, and Albert J. Menkveld, 2009, Does algorithmic trading improve liquidity? Working paper, University of California, Berkeley.

Heston, Steven L., and Ronnie Sadka, 2008a, Seasonality in the cross-section of stock returns, Journal of Financial Economics 87, 418-445.

Heston, Steven L., and Ronnie Sadka, 2008b, Seasonality in the cross-section of stock returns: The international evidence, Forthcoming, Journal of Financial and Quantitative Analysis.

Holden, Craig W., and Avanidhar Subrahmanyam, 2002, News events, information acquisition, and serial correlation, Journal of Business 75, 1-32.

Hora, Merrell, 2006, Tactical liquidity trading and intraday volume, Working paper, Credit Suisse.

Huberman, Gur, and Werner Stanzl, 2005, Optimal Liquidity Trading, Review of Finance 9, 165200.

Hvidkjaer, Soeren, 2008, Small trades and the cross-section of stock returns, Review of Financial Studies 21, 1123-1151.

Jain, Prem C., and Gun-Ho Joh, 1988, The dependence between hourly prices and trading volume, Journal of Financial and Quantitative Analysis 23, 269-283.

Jegadeesh, Narasimhan, 1990, Evidence of predictable behavior of security returns, Journal of Finance 45, 881-898.

Jegadeesh, Narasimhan, and Sheridan Titman, 1993, Returns to buying winners and selling losers: Implications for stock market efficiency. Journal of Finance 48, 65-91. 
Jeria, David, and George Sofianos, 2008, Algo evolution: From 4Cast to OptimIS and lower shortfall, Street Smart 37, 1-5.

Kamstra, Mark J., Lisa A. Kramer, and Maurice D. Levi, 2003, Winter blues: Seasonal affective disorder (SAD) stock market returns, American Economic Review 93, 324-343.

Keim, Donald B., 1983, Size-related anomalies and stock return seasonality: Further evidence, Journal of Financial Economics 12, 13-32.

Keim, Donald B., 1989, Trading patterns, bid-ask spreads, and estimated security returns: The case of common stocks at calendar turning points, Journal of Financial Economics 25, 75-97.

Korajczyk, Robert A., and Ronnie Sadka, 2004, Are momentum profits robust to trading costs? Journal of Finance 59, 1039-1082.

Korajczyk, Robert A., and Ronnie Sadka, 2008, Pricing the commonality across alternative measures of liquidity, Journal of Financial Economics 87, 45-72.

Large, Jeremy, 2007, Measuring the resiliency of an electronic limit order book, Journal of Financial Markets 10, 1-25.

Lee, Charles M. C., and Mark J. Ready, 1991, Inferring trade direction from intraday data, Journal of Finance 46, 733-754.

Lehmann, Bruce, 1990, Fads, martingales and market efficiency, Quarterly Journal of Economics $105,1-28$.

Lehmann, Bruce N., and David M. Modest, 2005, Diversification and the optimal construction of basis portfolios, Management Science 51, 581-598.

Lo, Andrew W., and A. Craig MacKinlay, 1990, When are contrarian profits due to stock market overreaction? Review of Financial Studies 3, 175-208.

Lo, Andrew W., and Jiang Wang, 2006, Trading volume: Implications of an intertemporal capital asset pricing model, Journal of Finance 61, 2805-2840.

Lou, Dong, 2008, A flow-based explanation for return predictability, Working paper, Yale University.

Pagano, Michael S., Lin Peng, and Robert A. Schwartz, 2008, The quality of price formation at market openings and closings: Evidence from the Nasdaq stock market, Working paper, Villanova University.

Roll, Richard, 1984, A simple implicit measure of the effective bid-ask spread in an efficient market, Journal of Finance 39, 1127-1139.

Rozeff, Michael S., and William R. Kinney, 1976, Capital market seasonality: The case of stock returns, Journal of Financial Economics 3, 379-402.

Sadka, Ronnie, 2006, Momentum and post-earnings-announcement drift anomalies: The role of liquidity risk, Journal of Financial Economics 80, 309-349.

Stoll, Hans R., 1978, The supply of dealer services in securities markets, Journal of Finance 33, 1133-1151.

Tversky, Amos, and Daniel Kahneman, 1974, Judgment under uncertainty: Heuristics and biases, Science 185, 1124-1131.

Vayanos, Dimitri, 2001, Strategic trading in a dynamic noisy market, Journal of Finance 56, 131171.

Vayanos, Dimitri, and Paul Woolley, 2008, An institutional theory of momentum and reversal, Working paper, London School of Economics.

Wood, Robert A., Thomas H. McInish, and J. Keith Ord, 1985, An investigation of transactions data for NYSE stocks, Journal of Finance 40, 723-739. 


\section{Footnotes}

1. Following Fama (1976), these responses have the interpretation of excess returns on costless portfolios that have excess returns of $100 \%$ in a previous half-hour interval.

2. We use Fama and MacBeth (1973) t-statistics, which assume no autocorrelation in the coefficients of the cross-sectional regressions, because we find no evidence of significant autocorrelation in the estimates, $\widehat{\gamma}_{k}$.

3. The Internet Appendix is available on the Journal of Finance web site, at http://www.afajof.org/supplements.asp.

4. The returns on the separate winners and losers portfolios are available in the Internet Appendix (Table IA.II).

5. An $8 \%$ equity risk premium corresponds to approximately 3.2 basis points per trading day (that is, 800/250).

6. Korajczyk and Sadka (2004), Goyenko, Holden, and Trzcinka (2009), and Hasbrouck (2009) find larger quoted spreads. However, these papers have sample periods that include both pre-decimalization data and some include less liquid stocks.

7. In the Internet Appendix (Table IA.XI) we estimate the return spread for S\&P 500 stocks (which is the sample studied in Gurliacci, et al.) to be 2.19 basis points.

8. In the Internet Appendix (Figure IA.3) we present a version of Figure 4 that uses different scaling of the return results, which makes it easier to discern the return patterns for the mid-day period.

9. The estimates, $\widehat{g}_{t, k}$, do not display substantial autocorrelation so we do not make adjustments to the Fama-MacBeth standard errors.

10. Given the increased transactions volume in our sample period relative to that in Lee and Ready (1991), we do not require their five second minimum period between quotes and transactions. 